\title{
Fibroblast Growth Factor 21 (FGF21), Free Fatty Acid (FFA), High Sensitivity C-reactive Protein (hsCRP) and Homeostasis Model Assessment of Insulin Resistance (HOMA-IR) Among Indonesian Obese Non-Diabetic Males
}

\author{
Yani Lina ${ }^{1,2}$, Gatot Susilo Lawrence ${ }^{3}$, Andi Wijaya ${ }^{1,2}$, Suryani As'ad ${ }^{1}$
}

\section{Abstract}

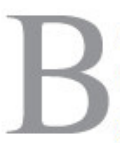

ACKGROUND: Fibroblast growth factor-21 (FGF21) is known as an important endocrine and paracrine regulator of metabolic homeostasis. Recent studies have shown that FGF21 attenuates lipolysis in human adipocytes, which is suggested as a FGF21's mechanism as anti-hyperlipidemia, anti-hyperglycemia and anti-obesity. The aim of this study was to measure the correlation between FGF21, FFA, hsCRP and HOMA-IR among Indonesian obese non diabetic males.

Method : This study was observational with cross sectional design. The analysis was done in 137 subjects aged 30-60 years with non diabetic abdominal obesity. We measured the biochemical markers FGF21, FFA, hsCRP, fasting insulin and fasting glucose. We also measured weight, height, waist circumference (WC), creatinine, serum glutamic oxaloacetic transaminase (SGOT), and serum glutamic pyruvic transaminase (SGPT), systolic blood pressure (SBP) and diastolic blood pressure (DBP). Correlation between markers was measured using Pearson and Spearman's analysis.

RESULT: There were significant positive correlations between FGF21-HOMA-IR ( $\mathrm{r}=0.314$, $\mathrm{p}=0.000$ ); FGF21WC $(\mathrm{r}=0.173, \mathrm{p}=0.043)$; FFA-hsCRP $(\mathrm{r}=0.256, \mathrm{p}=$ 0.003); FFA-WC $(\mathrm{r}=0.281, \mathrm{p}=0.001)$; WC-hsCRP ( $\mathrm{r}$ $=0.270, \mathrm{p}=0.001)$; and WC-HOMA-IR $(\mathrm{r}=0.279, \mathrm{p}$
$=0.001)$. There was insignificant negative correlation between FGF21-FFA ( $\mathrm{r}=-0.038, \mathrm{p}=0.657)$ and FGF21$\mathrm{hsCRP}(\mathrm{r}=-0.061, \mathrm{p}=0.482)$.

Conclusion : In this study we found that although there was no significant correlation, FGF21 might act as an antilipolytic and anti-inflammation agent among Indonesian obese non-diabetic males. Our findings agree with results of previous studies that the positive correlation between FGF21-WC and FGF21-HOMA-IR might occur as a compensatory mechanism or resistance to FGF21 in obesity.

KEYWORDS: Obesity, FGF21, FFA, hsCRP, HOMAIR.

\section{Introduction}

The human genome encodes 22 members of the fibroblast growth factor (FGF) family. Most function in diverse processes such as development and wound healing. But three members-FGF19 (FGF15 in the mouse),FGF21, and FGF23 - have recently emerged as metabolic hormones.1

Two studies (Badman et al., 2007; Inagaki et al., 2007) demonstrate that FGF21, a circulating protein produced in the liver in response to the PPAR $\alpha$ transcription factor, is a "missing link" in the biology of fasting, inducing adipose tissue lipolysis, liver ketogenesis, and metabolic adaptation 
to the fasting state. FGF21 actions on liver are ascribed as endocrine but could also be paracrine. Fasting is detected by the brain, leading to lipolysis and contributing to other adaptations such as torpor. Fatty acids are released from adipose tissue, taken up by the liver, and either oxidized or converted to ketones. Ketones released by the liver are used as fuel by the brain. Activation of PPAR $\alpha$, presumably via activation by fatty acids, increases transcription of FGF21. FGF21 contributes to ketogenesis in liver, lipolysis in adipose, and adaptation such as torpor by the brain.2

On the contrary, Arner's study showed that FGF21 attenuates hormone-stimulated lipolysis in both human and murine adipocytes. In vivo this would trigger a reduction of NEFA release and could thereby contribute to the insulin sensitizing effect of FGF21. The decrease in lipolysis could also contribute to a reduced lipotoxicity on islet cells in addition to the direct effects of FGF21 recently shown in beta cells. 3

These contradictions inspired us to study the correlation between FGF21, lipolysis, inflammation and insulin resistance. In this study, we aimed to find the correlation between FGF21, FFA, hsCRP and HOMA-IR among Indonesian obese non-diabetic males.

\section{Methods}

We used a cross sectional study with 137 obese male volunteers. Data collection was commenced in January 2009 and completed in June 2009. The study proposal was approved by the Health Research Ethics Committee of the Faculty of Medicine, University of Hasanuddin, Makassar, Indonesia.

The study subjects were screened from individuals coming to Prodia Clinical Laboratory in Jakarta for general medical check-up, employees and relatives of Prodia Clinical Laboratory in Jakarta. Subjects who were apparently healthy, male, aged $30-60$ years with WC $>90 \mathrm{~cm}$, and willing to follow the study protocol, were considered eligible to be included in the study.

Subjects who were consuming steroids; or had consumed antioxidant supplements such as vitamin C and/or E, green tea or other supplements classified as antioxidant in the last 3 weeks; or had been treated with anti-inflammatory drugs such as statins or thiazolidinedione in the last 3 weeks; or had liver dysfunction, kidney dysfunction, asthma, fever, and other acute inflammation were excluded from the study. Each subject was given explanation about the study and asked to sign informed consent prior to the commencement of the study.

All subjects were assessed after overnight fasting for at least 10 hours. The parameters of anthropometric measurements (height, weight, BMI, waist circumference, and blood pressure) and the biochemical variables (fasting glucose, fasting insulin, creatinine, SGOT, SGPT, FFA, FGF21, and hsCRP) were measured on all subjects. Insulin resistance was estimated using the homeostasis model assessment index (HOMA-IR), calculated as fasting glucose (in $\mathrm{mmol} / \mathrm{L}$ ) times fasting insulin (in $\mathrm{mIU} / \mathrm{L}$ ) divided by 22.5 .

FFA was measured with optimized enzymatic colorimetric assay for determination of free fatty acids (= Non-Esterified Fatty Acids, NEFA) (Roche Diagnostics $\mathrm{GmbH}$, Germany). The concentration of CRP was determined using a high-sensitivity chemiluminescent assay of Immulite ${ }^{\circledR}$ (Dade-Behring, Los Angeles, CA, USA). Fasting insulin was measured using ultrasensitive insulin ELISA of Mercodia. FGF21 was measured using an enzyme-linked immunoassay (ELISA) kit of Biovendor.

All statistical calculations were performed with the SPSS 11.5 statistical software package. Distributions of continuous variables were assessed for normality using the Kolmogorov-Smirnov. We used the Pearson or Spearman's rho correlation coefficient to assess the association between various anthropometric and biochemical variables. All tests were two-sided and considered significant at $\mathrm{p}<0.05$.

\section{Results}

The details of anthropometric measurements and biochemical variables are shown in Table 1 . Results of the Pearson and Spearman's correlation analysis are shown in Table 2 . We consider $\mathrm{p}<0.05$ as significant correlation.

Figure 1 shows a scattered graph between FGF21 and FFA; Figure 2 shows a scattered graph between FGF21 and hsCRP; Figure 3 shows a scattered graph between FGF21 and HOMA. 
Table 1. Results of Anthropometric Measurement and Biochemical Variables.

\begin{tabular}{|l|c|c|c|c|c|}
\hline & $\mathbf{n}$ & Minimum & Maximum & Mean & SD \\
\hline Age $($ years $)$ & 137 & 30.00 & 56.00 & 38.49 & 5.66 \\
\hline SBP $(\mathrm{mmHg})$ & 137 & 90.00 & 150.00 & 114.93 & 13.45 \\
\hline DBP $(\mathrm{mmHg})$ & 137 & 60.00 & 110.00 & 76.28 & 9.93 \\
\hline Height $(\mathrm{cm})$ & 137 & 156.00 & 185.00 & 166.15 & 5.21 \\
\hline Weight $(\mathrm{kg})$ & 137 & 65.00 & 120.00 & 79.68 & 7.98 \\
\hline WC $(\mathrm{cm})$ & 137 & 91.00 & 126.00 & 97.30 & 5.57 \\
\hline hSCRP $(\mathrm{mg} / \mathrm{L})$ & 137 & 0.22 & 9.12 & 2.24 & 1.83 \\
\hline SGOT $(\mathrm{U} / \mathrm{L})$ & 137 & 16.00 & 60.00 & 29.04 & 8.18 \\
\hline SGPT $(\mathrm{U} / \mathrm{L})$ & 137 & 14.00 & 84.00 & 37.53 & 14.22 \\
\hline FPG $(\mathrm{mg} / \mathrm{dL})$ & 137 & 74.00 & 119.00 & 90.08 & 8.77 \\
\hline Creatinine $(\mathrm{mg} / \mathrm{dL})$ & 137 & 0.60 & 1.40 & 1.09 & 0.16 \\
\hline FFA $(\mathrm{mM})$ & 137 & 0.18 & 1.12 & 0.58 & 0.19 \\
\hline FGF21 $(\mathrm{pg} / \mathrm{mL})$ & 137 & 7.00 & 670.19 & 217.34 & 130.20 \\
\hline HOMA-IR & 137 & 0.43 & 16.64 & 2.17 & 1.95 \\
\hline
\end{tabular}

FGP+ Fasting Plasma Glucose

Table 2. Pearson and Spearman's Correlation Analysis

\begin{tabular}{|c|c|c|c|c|c|}
\hline & wc & hsCRP & FFA & FGF21 & HOMA-IR \\
\hline WC (r) & 1 & $0.270^{\star \star}$ & $0.281^{\star \star}$ & $0.173^{*}$ & $0.279^{\star \star}$ \\
\hline (p) & - & 0.001 & 0.001 & 0.0431 & 0.001 \\
\hline hsCRP (r) & $0.270^{\star \star}$ & 1 & $0.256^{\star \star}$ & -0.061 & -0.079 \\
\hline (p) & 0.001 & - & 0.003 & 0.482 & 0.357 \\
\hline FFA (r) & $0.281^{\star \star \star}$ & $0.256^{\star \star}$ & 1 & -0.038 & -0.062 \\
\hline (p) & 0.001 & 0.003 & - & 0.657 & 0.469 \\
\hline FGF21 (r) & $0.173^{\star}$ & -0.061 & -0.038 & 1 & $0.314^{\star \star}$ \\
\hline$(p)$ & 0.043 & 0.482 & 0.657 & - & 0.000 \\
\hline HOMA-IR (r) & $0.279^{\star \star}$ & -0.079 & -0.062 & $0.314^{\star \star}$ & 1 \\
\hline (p) & 0.001 & 0.357 & 0.469 & 0.000 & - \\
\hline
\end{tabular}




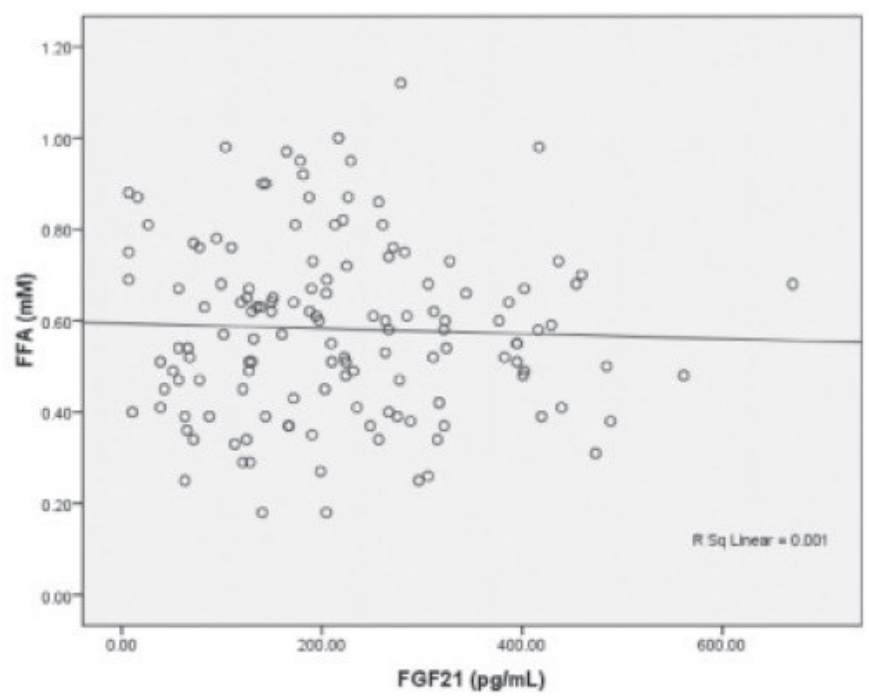

Figure 1. Scatter Analysis between FGF21 and FFA

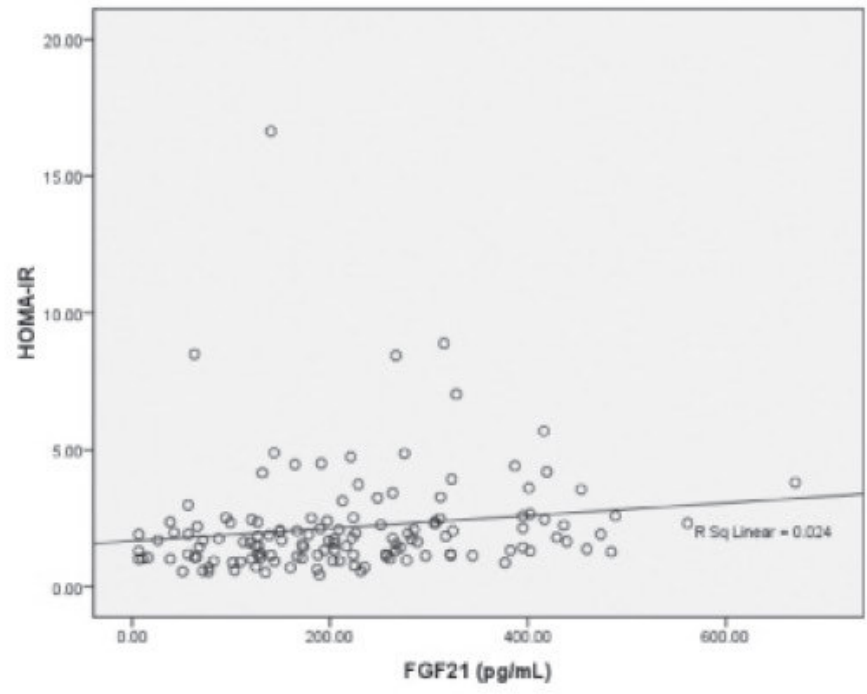

Figure 2. Scatter Analysis between FGF21 and HOMA-IR

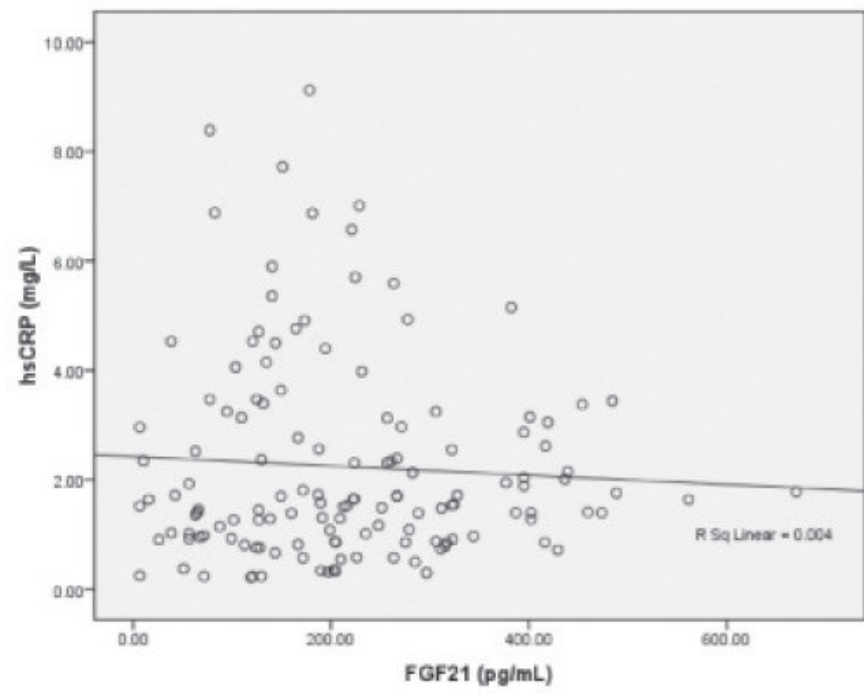

Figure 3. Scatter Analysis between FGF21 and hsCRP 


\section{Discussion}

In this study, it was shown that obesity (especially central obesity characterized by $\mathrm{WC}>90 \mathrm{~cm}$ ) was closely related to inflammation and insulin resistance (WC had a significant positive correlation with hsCRP and HOMA-IR). Our study also showed that obesity was related to increased lipolysis (WC had a significant positive correlation with FFA). Our study confirmed some previous studies that obesity was related to insulin resistance and inflammation through increased FFA concentration.

An expanding fat mass releases increasing amounts of compounds such as FFA, angiotensin 2 , resistin, TNF- $\alpha$, interleukin 6, interleukin $1-\beta$ and others. Plasma FFA levels are usually elevated in obesity because the enlarged adipose tissue mass releases more FFA and FFA clearance may be reduced. Moreover, once plasma FFA levels are elevated, they will inhibit insulin's anti-lipolytic action, which will further increase the rate of FFA release into the circulation. FFA is known mediate insulin resistance (through decreasing tyrosine phosphorylation of the insulin receptor substrate-1 (IRS-1)) and inflammation (through activation of proinflammatory $\mathrm{NF} x \mathrm{~B}$ pathway resulting in increased hepatic expression of several proinflammatory cytokines). 4

In this study, FGF21 had a significant positive correlation with WC and HOMA-IR. Result of our study confirmed that of Arner's study that has shown that serum FGF21 levels were significantly elevated in overweight/ obese subjects and correlated positively with fasting insulin and insulin resistance index (HOMA-IR).

FGF21 was recently known as a secreted protein expressed by different organs and tissues including adipose tissue, liver, pancreas, and muscle and improves glucose tolerance. Thus, FGF21 significantly stimulates glucose uptake in 3T3-L1 fat cells. Furthermore, FGF21 improves pancreatic $\beta$-cell function and survival by activation of p44/42 mitogen activated protein kinase. The paradoxical increase of serum FGF21 in obesity-related disorders might be a defensive response of the human body to counteract the metabolic stress imposed by obesity. Alternatively, obesity may cause resistance to FGF21 actions, leading to its compensatory upregulation.5,6
According to our study, FGF21 has insignificant negative correlation with FFA and hsCRP. We suggest that the increase of serum FGF21 in obesity might be a compensatory upregulation of FGF21 to improve insulin sensitivity and inflammation through negative correlation with FFA. We suggest that among Indonesian obese non diabetic males, FGF21 acts as an antilipolytic that suppresses lipolysis, therefore, reduction of FFA concentration will improve insulin sensitivity and inflammation among obese non diabetic males.

\section{Conclusions}

According to our study, we can conclude that although there is no significant correlation, FGF21 may act as an antilipolytic and anti-inflammation agent among Indonesian obese non diabetic males. We also have confirmed some previous studies that the positive correlation between FGF21-WC and FGF21-HOMA-IR might occur as a compensatory mechanism or resistance to FGF21 in obesity.

\section{Acknowledgements:}

We thank the Prodia Foundation for Research and Training for the invaluable support to this study.

\section{References:}

1. Moore DD. Sister Act. Science 2007; 316 : 1436-1438

2. Reitman ML. FGF21 : A Missing Link in the Biology of Fasting. Cell Metab 2007; $5:$ 405-407

3. Arner P, Pettersson A, Mitchell PJ, Dunbar JD, Kharitonenkov A, Ryden M. FGF21 Attenuates Lipolysis in Human Adipocytes - A Possible Link to Improved Insulin Sensitivity. FEBS Letters 2008; 582: 1725-1730

4. Boden G. Obesity and Free Fatty Acids (FFA). Endocrinol Metab Clin North Am 2008; 37 : 635-646

5. Stein S, Stepan H, Kratzsch J, Verlohren M, Verlohren HJ, Drynda K, et al. Serum Fibroblast Growth Factor 21 Levels in Gestational Diabetes Mellitus in Relation to Insulin Resistance and Dyslipidemia. Metabolism 2010; $59: 33-37$

6. Zhang X, Yeung DCY, Karpisek M, Stejskal D, Zhou ZG, Liu $F$, et al. Serum FGF21 Levels Are Increased in Obesity and Are Independently Associated With the Metabolic Syndrome in Humans. Diabetes 2008; 57: 1246-1253 